\title{
Article \\ How Poloxamer Addition in Hyaluronic-Acid-Decorated Biodegradable Microparticles Affects Polymer Degradation and Protein Release Kinetics
}

\author{
Teresa Silvestri ${ }^{1}$, Barbara Immirzi ${ }^{2}$, Giovanni Dal Poggetto ${ }^{2} \mathbb{D}$, Paola Di Donato ${ }^{3,4}$, Valentina Mollo ${ }^{5}$, \\ Laura Mayol 1,6,*(D) and Marco Biondi 1,6
}

\section{check for} updates

Citation: Silvestri, T.; Immirzi, B.; Dal Poggetto, G.; Di Donato, P.; Mollo, V.; Mayol, L.; Biondi, M. How Poloxamer Addition in

Hyaluronic-Acid-Decorated Biodegradable Microparticles Affects Polymer Degradation and Protein Release Kinetics. Appl. Sci. 2021, 11, 7567. https://doi.org/10.3390/ app11167567

Academic Editor: David G. Calatayud

Received: 2 July 2021

Accepted: 14 August 2021

Published: 18 August 2021

Publisher's Note: MDPI stays neutral with regard to jurisdictional claims in published maps and institutional affiliations.

Copyright: (c) 2021 by the authors. Licensee MDPI, Basel, Switzerland. This article is an open access article distributed under the terms and conditions of the Creative Commons Attribution (CC BY) license (https:/ / creativecommons.org/licenses/by/ $4.0 /)$.
1 Scuola di Medicina e Chirurgia, Università di Napoli Federico II, Via Domenico Montesano 49, 80131 Napoli, Italy; teresa.silvestri@unina.it (T.S.); mabiondi@unina.it (M.B.)

2 Institute of Polymers, Composites and Biomaterials (IPCB), C/o Comprensorio Olivetti, Via Campi Flegrei 34 80078 Pozzuoli, Italy; barbara.immirzi@ipcb.cnr.it (B.I.); giovanni.dalpoggetto@ipcb.cnr.it (G.D.P.)

3 Department of Science and Technology, University of Naples Parthenope, Centro Direzionale, Isola C4, 80143 Naples, Italy; paola.didonato@uniparthenope.it

4 Institute of Biomolecular Chemistry, National Research Council of Italy, Via Campi Flegrei 34, 80078 Pozzuoli, Italy

5 Italian Institute of Technology@CRIB Center for Advanced Biomaterials for Health Care, 80125 Napoli, Italy; Valentina.Mollo@iit.it

6 Centro di Ricerca Interdipartimentale sui Biomateriali (CRIB), Università di Napoli Federico II, Piazzale Tecchio 80, 80125 Napoli, Italy

* Correspondence: laumayol@unina.it

Abstract: Polymeric microparticles (MPs) designed for the intravitreal administration of therapeutic proteins result in a prolonged half-life in the vitreous and can delay or discourage the onset of adverse effects inevitably related to this route of administration. Hence, here we designed MPs composed of a polymeric blend based on poly(lactic-co-glycolic) acid and poloxamers, externally decorated with hyaluronic acid. The MPs are intended for intravitreal administration of bovine serum albumin. In detail, a systematic formulative study aiming to shed light on the complex relationship between protein release rate and MP degradation rate was carried out by means of calorimetric and gel permeation chromatography analyses. We found out that poloxamer addition caused a compact MP matrix, which led to a slight modification of the degradation kinetics and a reduction in the initial BSA initial release, which is of the utmost importance to ensure a relatively regular BSA release. It must also be underlined that for acid-labile molecules such as proteins, the poloxamer's presence induced complex and hardly predictable effects on MP degradation/protein release, due to the dynamic balance between the time-evolving hydrophilicity of MPs and the influence of poloxamers themselves on the PLGA degradation rate.

Keywords: biodegradable; microparticles; ocular drug delivery; PLGA; hyaluronic acid; poloxamers

\section{Introduction}

The bioavailability of drugs to the posterior segment of the eye is still a significant challenge. Indeed, the administration of active molecules by indirect routes, such as topical and systemic, is of very low effectiveness due to the presence of blood-ocular barriers. These cause poor drug penetration within the vitreous body and, therefore, a sub-therapeutic quantity of drug at the affected site [1]. For these reasons, intravitreal drug administration is still considered necessary for the successful treatment of posterior eye segment pathologies, as well as those related to neovascularization [2-4]. Indeed, the treatment of such disorders requires the administration of angiogenesis inhibitors, and only their intravitreal injection consistently enables the delivery of therapeutic agents in loco [5-7]. However, chronic intravitreal drug administration commonly elicits severe complications, such as retinal detachment, cataracts, hemorrhages and endophthalmitis, 
which are progressively more likely as the treatment progresses [8-10]. In this context, the reduction of the injection frequency allows us, in principle, to delay or discourage the onset of these adverse effects. Consequently, accomplishing a sustained drug administration strategy within the intravitreal space is more desirable than repeated injections [2].

From this standpoint, microparticles (MPs) designed for the intravitreal administration of therapeutic proteins lead to a prolonged half-life in the vitreous in a way that is not influenced by its clearance [11]. In two recent works, biodegradable MPs based on poly (lactic-co-glycolic) acid (PLGA), externally decorated with hyaluronic acid (HA), were produced $[12,13]$. In detail, the MPs were fabricated by a modified version of the doubleemulsion solvent evaporation technique using a PLGA/poloxamer (POLOX) polymeric blend in the organic phase of the emulsion. POLOX are amphiphilic block copolymers of ethylene oxide and propylene oxide monomers and were used to enhance the affinity between lipophilic PLGA and hydrophilic HA [12-15].

HA was chosen because it is a major constituent of the vitreous body of the eye, and this allows a high affinity between the MPs and the vitreous body [16]. Indeed, the presence of HA on the surfaces of MPs has been shown to discourage their free diffusion in simulated vitreous body (SVB) [13], which promises to prevent microcarriers from interfering with patient visus. Moreover, the produced MPs could sustain the release of bovine serum albumin (BSA), used as a model protein.

It is known that PLGA degradation, which occurs through random ester bond cleavage, is triggered by water intrusion and catalyzed by acids [16,17]. PLGA-based MPs undergo bulk erosion since water can penetrate the MP inner regions and the trapped acidic oligomers prompt further autocatalytic degradation of the polymer [18]. Indeed, autocatalysis strongly depends on the formulation approach and significantly affects protein release [19]. Furthermore, bulk degradation probably results in low $\mathrm{pH}$ values within MPs, which in turn may be detrimental for the biological activity of acid-labile moieties, such as proteins [20]. In this panorama, amphiphilic POLOX are expected to modify bulk PLGA degradation through multiple mechanisms. For example, they may interfere with the local $\mathrm{pH}$ drop, change the MP morphology, and/or induce MP plasticization. Importantly, the additive presence in PLGA-based MPs is expected to change the release kinetics and/or burst of the loaded molecule(s).

To the best of our knowledge, there is no systematic study that reports on the relationship between the release profiles of a protein from MPs and its polymeric composition, on which the degradation kinetics depend. Hence, we aim to shed light on the complex relationship between the protein release rate and MP degradation rate. For this purpose, different MP formulations were produced by a modified double-emulsion solvent evaporation technique $[12,13]$, at different PLGA/POLOX weight ratios and overall polymer concentrations in the organic phase of the emulsion. The obtained MPs were loaded with BSA and fully characterized for their technological characteristics, which were then correlated with the degradation kinetics by calorimetric and gel permeation chromatography (GPC) analyses.

\section{Materials and Methods}

\subsection{Materials}

Poly(D,L-lactide-co-glycolide) (PLGA) (Resomer ${ }^{\circledR}$ RG504H; lactide/glycolide ratio 50:50; inherent viscosity: $0.57 \mathrm{dL} \mathrm{g}^{-1}$ in chloroform at $25^{\circ} \mathrm{C}$ ) was purchased from Evonik (Essen, Germany). Hyaluronic acid (HA) with a molecular weight (MW) of $1600 \mathrm{kDa}$ was provided by DSM (Kaiseraugst, Switzerland). In this work, poloxamers (POLOX) (PEOa-PPOb-PEOa) F127 ( $\mathrm{a}=100$ and b = 65, GPC in THF Mw $13.8 \mathrm{kDa}, \mathrm{Mn} 10.1 \mathrm{kDa}$, intrinsic viscosity $0.19 \mathrm{dL} \mathrm{g}^{-1}$ in THF $\left.35^{\circ} \mathrm{C}\right)$ and $\mathrm{F} 68(\mathrm{a}=76$ and $\mathrm{b}=29$, GPC in THF Mw 9.6 kDa, Mn $8.8 \mathrm{kDa}$, intrinsic viscosity $0.15 \mathrm{dL} \mathrm{g}^{-1}$ in THF $35^{\circ} \mathrm{C}$ ) from Lutrol (Basf, Ludwigshafen, Germany) were employed. Bovine serum albumin (BSA), dichloromethane (DCM), sodium azide, agar, dibasic sodium phosphate $\left(\mathrm{Na}_{2} \mathrm{HPO}_{4}\right)$, potassium chloride 
$(\mathrm{KCl})$ and sodium chloride $(\mathrm{NaCl})$ were obtained from Sigma-Aldrich (Saint Louis, $\mathrm{MO}$, USA). All the chemical substances were employed without any previous purification.

\subsection{Microparticle Preparation}

MPs were obtained by a modified double-emulsion solvent evaporation technique, as described previously $[12,13]$, without any chemical reaction. In detail, an internal aqueous phase $\left(\mathrm{W}_{1}\right)(0.25 \mathrm{~mL}$ of BSA solution in phosphate buffer, $0.4 \% w / v)$ was emulsified with $2.5 \mathrm{~mL}$ of an organic phase $(\mathrm{O})$ composed of $20 \%$ or $24 \% w / v$ overall polymer concentration and different PLGA/POLOX weight ratios (90:10 or 70:30 $w / w)$ in DCM (as reported in Table 1). The obtained primary emulsion $\left(\mathrm{W}_{1} / \mathrm{O}\right)$ was further emulsified with $40 \mathrm{~mL}$ of an external aqueous phase $\left(\mathrm{W}_{2}\right)$ containing HA and POLOX $(0.75$ and $0.0375 \mathrm{mg} / \mathrm{mL}$, respectively) to obtain the final double emulsion $\left(\mathrm{W}_{1} / \mathrm{O} / \mathrm{W}_{2}\right)$. The system was emulsified by a highspeed homogenizer (Diax 900 equipped with a10G probe, Heidolph, Germany) at 11,000 rpm for two minutes, for both first and second emulsion. Afterwards, the organic solvent evaporation and MP hardening were achieved by magnetic stirring (MR $3001 \mathrm{~K}$, Heidolph, Germany) overnight at room temperature. Finally, MPs were collected, centrifuged (5000 rpm, $4{ }^{\circ} \mathrm{C}$; Universal 16R, Hettich Zentrifugen, Germany), washed three times with double-distilled water and lyophilized for $24 \mathrm{~h}\left(-80^{\circ} \mathrm{C}, 0.1 \mathrm{mbar}, 24 \mathrm{~h}\right.$; LyoQuest, Telstar, Japan). As a control, we used PLGA MPs with an organic phase composed of PLGA only $(20 \%$ or $24 \% w / v)$, while the external aqueous phase was obtained in the absence of HA.

Table 1. Composition and naming of microspheres.

\begin{tabular}{cccccc}
\hline $\begin{array}{c}\text { Formulation } \\
\text { Name }\end{array}$ & $\begin{array}{c}\text { Overall Polymeric } \\
\text { Concentration \% }\end{array}$ & $\begin{array}{c}\text { PLGA: } \\
\text { POLOX Ratio }\end{array}$ & PLGA, mg & F68, mg & F127, mg \\
\hline $20-100$ & 20 & $100: 0$ & 500 & 0 & 0 \\
$20-90$ & 20 & $90: 10$ & 450 & 25 & 25 \\
$20-70$ & 20 & $70: 30$ & 350 & 75 & 75 \\
$24-100$ & 24 & $100: 0$ & 600 & 0 & 0 \\
$24-90$ & 24 & $90: 10$ & 540 & 30 & 30 \\
$24-70$ & 24 & $70: 30$ & 420 & 90 & 90 \\
\hline
\end{tabular}

\subsection{Microparticle Size}

The produced MPs were analyzed for their mean diameter and size distribution by laser diffraction (Mastersizer 3000E, Malvern Panalytical, Malvern, UK). The tests were run by dispersing MPs in double-distilled water under continuous stirring at $1500 \mathrm{rpm}$. The results and the standard deviation were averaged on triplicate samples.

\subsection{Microparticle Yield and Protein Entrapment Efficiency}

MP yield was gravimetrically obtained from the entire mass of MPs recovered after lyophilization $\left(-80^{\circ} \mathrm{C}, 0.1 \mathrm{mbar}, 24 \mathrm{~h}\right.$; LyoQuest, Telstar, Japan), while the actual loading of BSA into the MPs was achieved by the Bradford assay [13]. Briefly, for each batch, $10 \mathrm{mg}$ of MPs was solubilized in $500 \mu \mathrm{L}$ of dichloromethane. Then, $200 \mu \mathrm{L}$ of PBS (pH = 7.4) was added and the suspension was centrifuged (10,000 rmp, $10 \mathrm{~min}$; MIKRO20; Hettich, Tuttlingen, Germany), and $35 \mu \mathrm{L}$ of the aqueous solution was withdrawn and diluted with $665 \mu \mathrm{L}$ of the Bradford reagent for $15 \mathrm{~min}$. Afterwards, BSA was quantified by a spectrophotometric assay (UV-1800, Shimadzu; $\lambda=595 \mathrm{~nm}$ ), employing a quartz cuvette (6030-UV, $\mathrm{d}=10 \mathrm{~mm}$, Hellma Analytics). The linearity of the response was measured in the $0.49-500 \mu \mathrm{g} / \mathrm{mL}$ BSA concentration range $\left(\mathrm{r}^{2}>0.96\right)$. Results are expressed as actual BSA loading (mg of loaded BSA per $100 \mathrm{mg}$ of MPs) and BSA encapsulation efficiency (ratio of actual and theoretical BSA loading rate $\times 100) \pm$ the standard deviation of the values collected from at least three different batches. 


\subsection{BSA Release Kinetics}

In vitro release profiles of BSA from PLGA-based MPs were evaluated in simulated vitreous body (SVB), which was prepared following a previously published method [21]. Briefly, agar was dissolved in hot distilled water $(0.4 \% w / v)$ and, afterwards, HA (1600 kDa) was added $(0.5 \% w / v)$, along with a few drops of sodium azide solution $(0.02 \% w / v)$ as a preservative. For each experiment, a dispersion containing $25 \mathrm{mg}$ of MPs in SVB (weight ratio $=1: 20$ ) was poured into a vial; then, $1 \mathrm{~mL}$ of $\mathrm{PBS}$ at $\mathrm{pH} 7.4$ was poured onto the gel and the system was incubated at $37^{\circ} \mathrm{C}$ in a thermostatic bath. At predetermined times, $500 \mu \mathrm{L}$ of supernatant was withdrawn and replaced with fresh PBS, while monitoring the $\mathrm{pH}$ of the release medium with litmus paper. BSA was quantified by the Bradford assay as described in Section 2.4. Results are expressed as fraction of BSA released \pm the standard deviation of three replicas.

The main phenomena dictating protein release were estimated by modeling the released BSA fraction through the equation proposed by Corrigan and Xue, adapted for the MPs produced in this work [22]. In brief, BSA release from PLGA-based MPs was considered to be mainly governed by two major mechanisms, namely solubilization and matrix erosion. The first refers to protein dissolution at the interface between MP porosities/surface and the release medium, which is primarily dictated by protein diffusion through aqueous domains within MP evolving macropores [23]. The second mechanism considers the fraction of released BSA controlled by matrix bulk erosion, which is due to PLGA degradation and POLOX solubilization.

The first term is defined by Equation (1):

$$
\varphi_{S}=\varphi_{S, \infty}\left[1-\exp \left(-k_{S} t\right)\right],
$$

where $\varphi_{S}$ is the fraction of BSA released due to solubilization at time $t$ [day]; $\varphi_{S, \infty}$, is BSA fraction released after a virtually infinite time if no other phenomena contribute to BSA release; $k_{S}\left[\right.$ day $\left.^{-1}\right]$ is the solubilization rate constant.

Erosion-controlled release refers to $\left(1-\varphi_{S}\right)$, which is the fraction of the protein entrapped within MPs, which is described by the following Equation (2):

$$
\varphi_{E}=\left(1-\varphi_{S}\right) \frac{\exp \left[k_{E}\left(t-t_{M A X}\right)\right]}{1+\exp \left[k_{E}\left(t-t_{M A X}\right)\right]} .
$$

Here, $k_{E}\left[\right.$ day $\left.^{-1}\right]$ is the erosion constant, which takes into account the loss of matter due to both PLGA autocatalytic degradation and POLOX dissolution; $t_{\text {MAX }}$ [day] is the time at maximum release rate. The sum of Equations (1) and (2) provides the total released BSA fraction. The fraction, therefore, is given by the sum of the contributions:

$$
\varphi_{\text {TOT }}=\varphi_{S, \infty}\left[1-\exp \left(-k_{S} t\right)\right]+\left(1-\varphi_{S}\right) \frac{\exp \left[k_{E}\left(t-t_{M A X}\right)\right]}{1+\exp \left[k_{E}\left(t-t_{M A X}\right)\right]}
$$

Equation (3) has been solved by nonlinear least squares fitting using a 4th-order Runge-Kutta method, with $\varphi_{S, \infty}, k_{S}, k_{E}$ and $t_{M A X}$ as adjustable parameters.

\subsection{Degradation Studies of $M P S$}

MPs were suspended in PBS $(0.1 \% w / v)$ and incubated at $37^{\circ} \mathrm{C}$ in a thermostatic bath. At scheduled time points $(7,14,21,28$ days), the samples were centrifuged (5000 rpm, 10 min. $4{ }^{\circ} \mathrm{C}$; Universal 16R, Hettich Zentrifugen, Germany) and the PBS supernatant, containing the solubilized MPs fractions, was withdrawn and replaced with an equal volume of fresh PBS. Then, the samples were lyophilized $\left(-80^{\circ} \mathrm{C}, 0.1 \mathrm{mbar}, 24 \mathrm{~h}\right.$; LyoQuest, Telstar, Japan). 


\subsection{Gel Permeation Chromatography (GPC)}

Gel permeation chromatography (GPC) allowed us to evaluate the molecular weight (Mw mass average molar mass and Mn number average molar mass) variation of the soluble polymers composing the MPs as a consequence of the release rate related to the degradation mechanism over time. Since only a fraction of MPs are soluble in tetrahydrofuran (THF,) as a first step, MPs were suspended in THF for $24 \mathrm{~h}$ at $40^{\circ} \mathrm{C}$ in order to solubilize PLGA and POLOX. The insoluble fraction, composed of BSA and HA, was removed by filtration (PTFE membranes of $0.22 \mu \mathrm{m}$ porosity), while the soluble phase was dried in order to estimate the exact mass of soluble polymers. Purified samples were redissolved in THF and analyzed using a GPC Max Viscotek system equipped with a TDA 305 detector (Refractive Index, Low Angle Light Scattering, Right Angle Light Scattering and Viscometer) and UV detector. Column set was composed of a pre-column Phenogel Phenomenex and two columns of $10^{3}$ and $50 \mathrm{~g} \cdot \mathrm{mol}^{-1}$, respectively. The $50 \mathrm{~g} \cdot \mathrm{mol}^{-1}$ column was selected to take into account the very-low-molecular-weight fraction (low exclusion limit is $100 \mathrm{Da}$ ). PLGA, POLOX and all the samples reported in Table 1 were dissolved at $2-4 \mathrm{mg} / \mathrm{mL}$ concentration and eluted in HPLC-grade THF (Romil) at $40{ }^{\circ} \mathrm{C}$. The injection volume was $100 \mu \mathrm{L}$, the flow rate $0.8 \mathrm{~mL} / \mathrm{min}$. The chosen method of analysis was universal calibration with PS standard ranging from $66 \mathrm{kDa}$ to $937 \mathrm{Da}$. The measurements, performed at $35^{\circ} \mathrm{C}$ according to the temperatures of columns and detectors, were performed for $35 \mathrm{~min}$ in duplicate.

\subsection{Thermodynamic Analysis by Differential Scanning Calorimetry (DSC)}

To determine how the glass transition of PLGA is affected by the release/degradation time and formulation variable, differential scanning calorimetry studies (DSC; TA Q20) were performed to identify the thermodynamic transitions of MPs during degradation. In particular, the MPs collected at predetermined incubation times were centrifuged, washed and lyophilized. Then, carefully weighed masses of each MP sample (2-5 mg) were placed in hermetically sealed aluminum capsules and subjected to a double dynamic scan $\left(10-80{ }^{\circ} \mathrm{C}\right.$; heating rate: $\left.5^{\circ} \mathrm{C} / \mathrm{min}\right)$ in nitrogen flow at $50.0 \mathrm{~mL} / \mathrm{min}$. Glass transition temperatures were obtained during the second scan as the inflection points of the thermograms. Melting points were obtained as the extrapolated onset points in the DSC endothermic peaks, where present.

\section{Results and Discussion}

\subsection{Microparticle Characterization}

The morphology of MPs was evaluated by scanning electron microscopy (SEM) (Figure 1). In all the produced formulations, spherical particles were obtained. Interestingly, the presence of POLOX in the organic phase of the emulsion, even in a low amount (0.5:0.5:9 F68:F127:PLGA weight ratio), had a fundamental impact on the resulting particle morphology. Indeed, the surface of POLOX-containing MP formulations was smooth and non-porous (Figure 1A,B,D,E), while evident surface pores were highlighted in the case of MPs consisting of PLGA only (Figure 1C,F). This is consistent with the results obtained in a previous publication [14], in which we showed that HA spontaneously arranged toward the $W_{2}$ phase, due to the amphiphilic nature of POLOX, which enhances the affinity between $\mathrm{W}_{2}$ and the $\mathrm{O}$ phase.

The mean diameter, BSA entrapment efficiency and yield of the obtained formulations are reported in the following Table 2.

Importantly, POLOX presence in the MP bulk had a major influence also on the particle sizes (Figure 1, Table 1). In actual fact, the diameter of MPs decreased in the presence of the poloxamers: the average diameter of the MPs composed of PLGA alone was around $30 \mu \mathrm{m}$, while in the presence of the poloxamers, MPs of 14-15 $\mu \mathrm{m}$ were obtained. Protein encapsulation efficiency (EE) was between 43 and $81 \%$ in all cases, as shown in Table 2. In particular, EE was found to be significantly higher when the overall polymer concentration in the organic phase was $24 \% w / v$. This result can be reasonably explained considering that the higher polymer concentration leads to an increase in the viscosity of 
the organic phase of the emulsion and therefore reduced shear stress during the emulsion. This can be reasonably associated with the slower diffusion of BSA towards the external aqueous phase [24] and, overall, the increased EE of the protein.
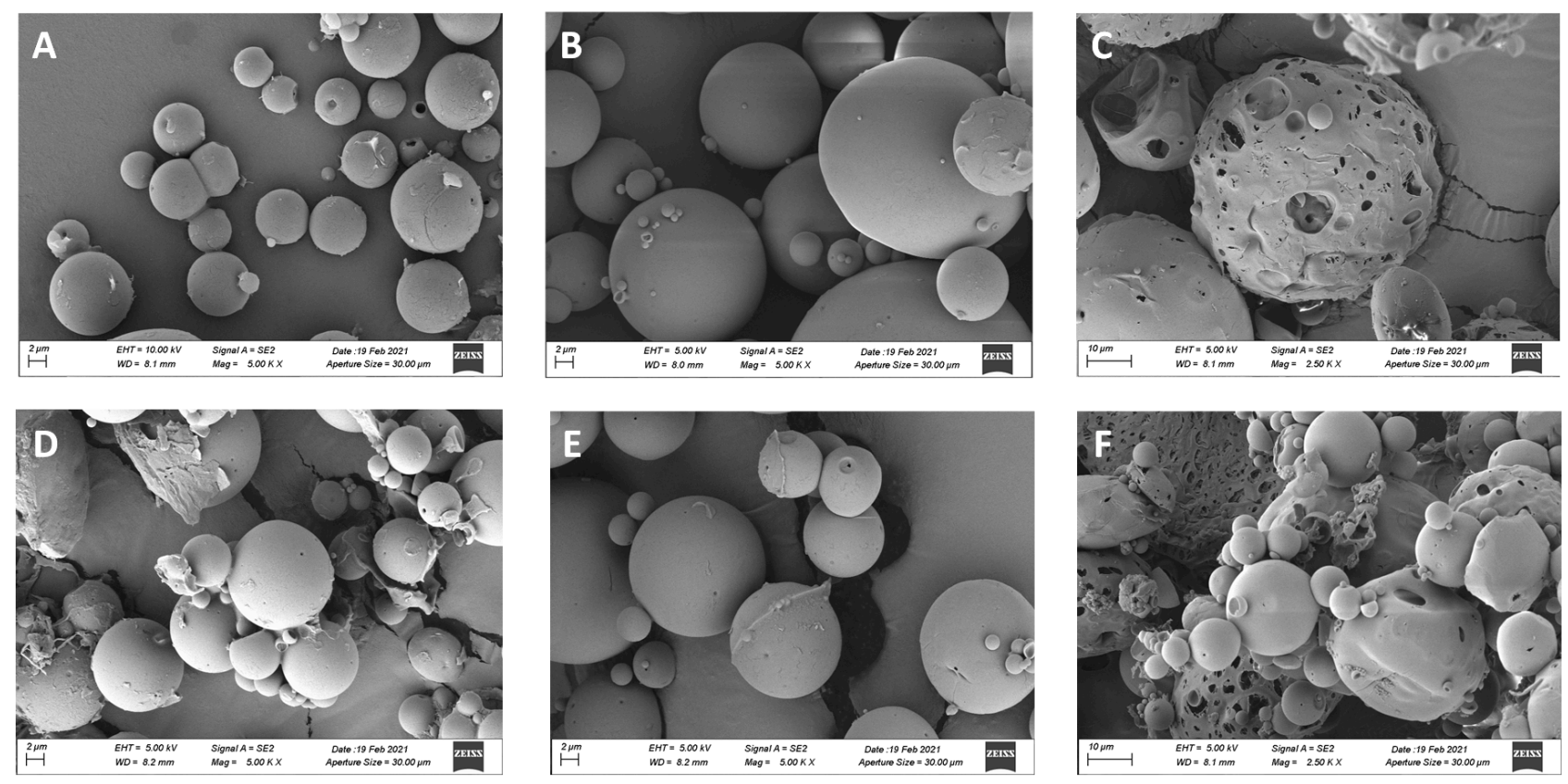

Figure 1. SEM images of (A) 20-70 (B) 20-90 and (C) 20-100 (D) 24-70 (E) 24-90 and (F) 24-100 MPs. The bar is $2 \mu \mathrm{m}$ in $(\mathbf{A}, \mathbf{B}, \mathbf{D}, \mathbf{E})$ and $10 \mu \mathrm{m}$ in $(\mathbf{C}, \mathbf{F})$.

Table 2. Overall technological features of the produced MPs.

\begin{tabular}{cccc}
\hline Formulation & Mean Diameter $(\boldsymbol{\mu m}) \mathbf{(} \pm$ SD) & E.E. $(\%)( \pm$ SD) & Yield $( \pm$ SD) \\
\hline $20-100$ & $30 \pm 8.4$ & $49 \pm 4$ & $27 \pm 12$ \\
$20-90$ & $14 \pm 6.0$ & $63 \pm 13$ & $38 \pm 10$ \\
$20-70$ & $14 \pm 6.0$ & $43 \pm 1.0$ & $43 \pm 24$ \\
$24-100$ & $31 \pm 5.0$ & $61 \pm 10$ & $30 \pm 15$ \\
$24-90$ & $15 \pm 7.0$ & $68 \pm 19$ & $35 \pm 21$ \\
$24-70$ & $15 \pm 6.2$ & $81 \pm 18$ & $27 \pm 15$ \\
\hline
\end{tabular}

\subsection{Release Kinetics of $B S A$ in $M P S$}

In vitro release profiles of BSA from MPs in SVB, along with the results of numerical simulations, are shown in Figures 2 and 3. When polymer concentration in the organic phase of the emulsion was $20 \% w / v$, the presence of POLOX influenced the protein release kinetics. Indeed, the 20-100 formulation showed considerable initial release, which can be attributed to the porous surface of the MPs. The residual protein discharge was sustained for 4 weeks. In the case of 20-90 MPs, BSA was released in a similar time, but with a very attenuated initial release. Protein release from the 20-70 MPs was found to be unsatisfactorily reproducible, as indicated by the high standard deviations.

Likewise, the BSA release profiles from the 24-90 and 24-100 formulations were essentially similar, with complete delivery within 4 weeks, while, in the case of the 24-70 formulation, protein discharge was slightly quicker. Interestingly, no substantial initial was detected for the 24-70, 24-90 and 24-100 formulations, while a moderate acceleration of protein release was seen between 7 and 14 days, which implies that considerable fractions of bulk BSA were present for these formulations in the specified timeframe. 

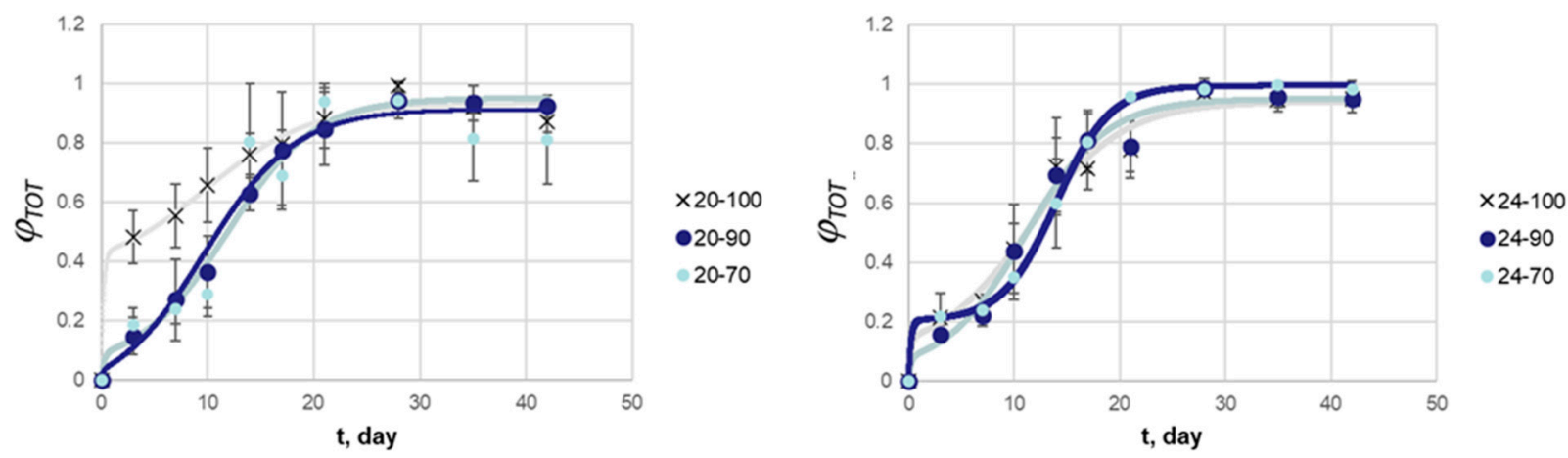

Figure 2. In vitro protein release kinetics from PLGA-based microparticles at $20 \%$ (left) and $24 \%$ (right) $w / v$ overall polymer content in the organic phase of the emulsion. Solid lines represent numerical simulations.
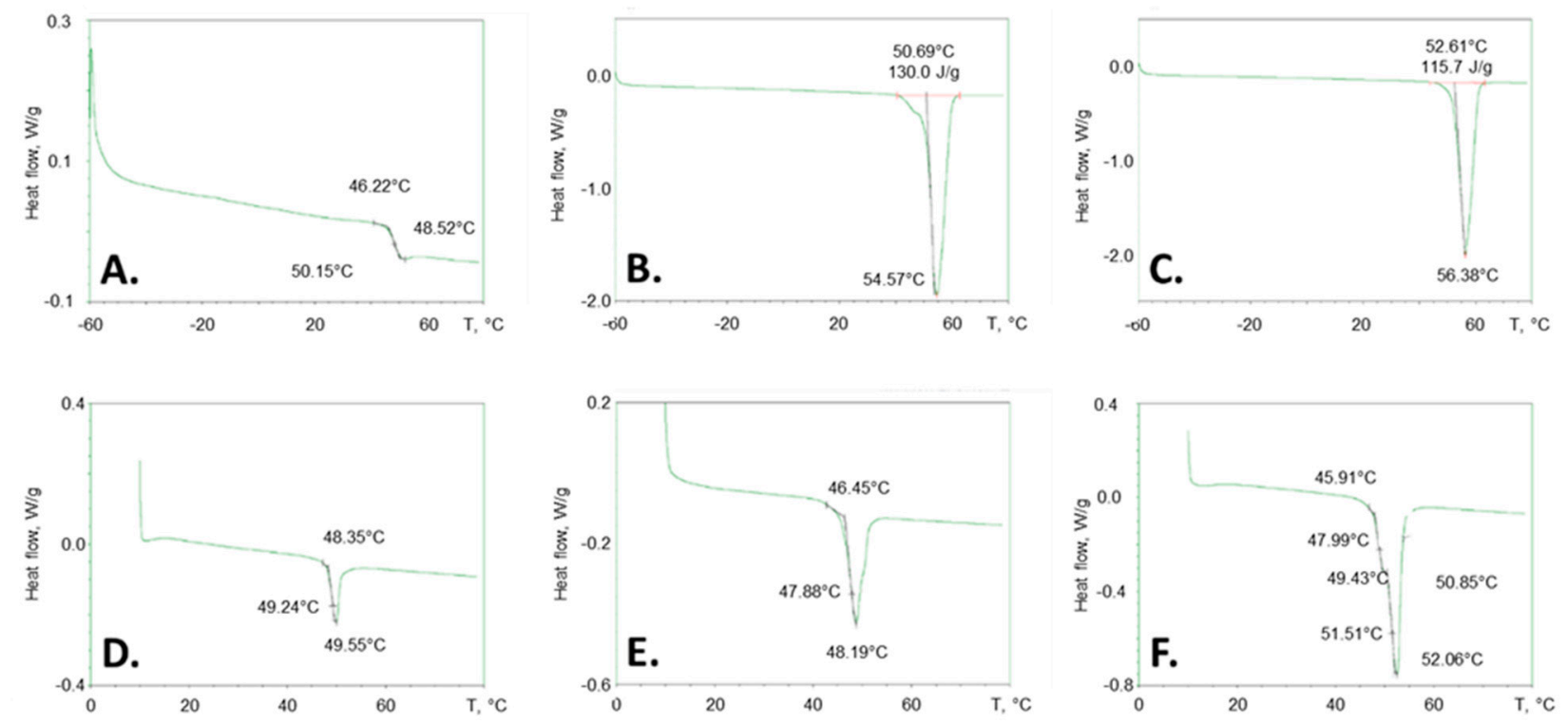

Figure 3. Representative DSC thermograms of PLGA powder (A); F68 powder (B); F127 powder (C); $20-100$ (D), $20-90$ (E), 20-70 (F) microparticles. Exotherm is directed upward.

The released fraction was modeled as described in Section 2.5 and the best fit parameters are summarized in Table 3 . The values of the solubilization rate constant $k_{S}$ were relatively close for all formulations, which indicates that superficial phenomena within MPs during release are comparable irrespective of the polymer concentration and POLOX presence.

Table 3. Best fit results of BSA release kinetics modelling for the six MP formulations.

\begin{tabular}{ccccccc}
\hline & $\mathbf{2 0 - 7 0}$ & $\mathbf{2 0 - 9 0}$ & $\mathbf{2 0 - 1 0 0}$ & $\mathbf{2 4 - 7 0}$ & $\mathbf{2 4 - 9 0}$ & $\mathbf{2 4 - 1 0 0}$ \\
\hline$k_{S}\left[\right.$ day $\left.^{-1}\right]$ & 4.60 & 4.41 & 4.86 & 4.40 & 4.33 & 4.33 \\
$\varphi_{S, \infty}$ & 0.031 & 0.089 & 0.429 & 0.204 & 0.080 & 0.146 \\
$k_{E}\left[\right.$ day $\left.^{-1}\right]$ & 0.234 & 0.241 & 0.202 & 0.391 & 0.262 & 0.229 \\
$t_{M A X}[$ day $]$ & 9.78 & 11.7 & 9.89 & 14.0 & 11.0 & 11.5 \\
\hline
\end{tabular}

When the polymer concentration was $20 \% w / v$ in the organic phase of the emulsion, $\varphi_{S, \infty}$ increased with increasing PLGA:POLOX ratio. This is related to the extent of the initial BSA release and consistent with the strong initial release observed in the 20-100 formulation. As for MPs produced with a $24 \% w / v$ polymer concentration in the organic phase of the emulsion, no clear trend of $\varphi_{S, \infty}$ with POLOX percentage was seen, indicating that the 
dense MP matrix associated with a high polymer concentration effectively hampers BSA diffusion and subsequent release.

It should also be emphasized that POLOX had two contrasting effects on MP degradation/erosion. On the one hand, they increased the hydrophilicity of MPs, favoring their erosion; on the other hand, they diluted the PLGA, reducing the autocatalytic effects and therefore hindering erosion. The $k_{E}$ values of the best fit indicate that for 20-70 and 20-90 MPs, the two effects were roughly balanced. In the case of 24-70, 24-90 and 24-100 MPs, on the contrary, $k_{E}$ values descended as the percentage of PLGA increased. This indicates that, at the highest overall concentration of polymers, the effect of the promoted solubilization induced by POLOX prevailed in determining the erosion of MPs.

The $t_{M A X}$ values were around 10-12 days for all formulations, while being higher in the case of 24-70 MPs (14.0 days). In this latter case, the higher erosion rate described by $k_{E}$ is associated with a longer time, corresponding to the maximum erosion rate.

\subsection{DSC}

The thermograms of raw PLGA, POLOX and the produced MPs are summarized in Figure 3. As shown in Figure 3A, the thermogram of PLGA shows a glass transition temperature (Tg) of around $48.5^{\circ} \mathrm{C}$, while F68 and F127 POLOX exhibited an endothermic melting peak at approximately 54 and $56^{\circ} \mathrm{C}$ (Figure 3B,C). DSC traces of HA showed no thermodynamic events in the test conditions (data not shown). Similarly, MPs did not show the endothermic peaks of POLOX, thereby indicating that they were molecularly mixed with PLGA.

Figure 3 shows the representative DSC traces of the pure materials and of the asproduced 20-100, 20-90 and 20-70 MPs. HA did not show any thermal event in the utilized temperature range (data not shown). PLGA showed only a glass transition at around $48.5^{\circ} \mathrm{C}$ (Figure 3A), with no crystallinity or fusion due to its amorphous nature, while F68 and F 127 POLOX evidenced a relatively sharp endothermic peak attributed to polymer melting (Figure 3B,C, respectively). Interestingly, the thermograms of MPs (Figure 3D-F) displayed a glass transition event at temperatures similar to the Tg of pure PLGA. In all cases, anyhow, the glass transition event was overlapped with an endothermic peak attributable to POLOX. It must be underlined that 20-100 MPs were produced with POLOX in the external water phase; therefore, the small endothermic peak observed is probably due to the POLOX adhering to the external MP surface. As for the 20-90 and 20-70 formulations, the endothermic peak is more relevant due to the increasing presence of POLOX in the organic phase of the emulsion. These outcomes hint at the formation of a partially phase-separated matrix in the MPs. The same outcomes were found for the 24-100, 24-90 and 24-70 formulations.

Figure 4 depicts the time trend of $\mathrm{Tg}$ for all formulations, until day 28. The glass transition temperature was found to follow a slowly decreasing time trend, substantially superimposable for all MP formulations. Tg steadily decreased until day 28, dropping from approximately 49 to $42{ }^{\circ} \mathrm{C}$, due to the drop in the PLGA's molecular weight during release.

\subsection{GPC Analysis}

Supplementary Table S1 reports the molecular weight distributions, intrinsic viscosity (IV) and weight fraction of the polymeric part, named peak 1, ranging from 9 to $15 \mathrm{~min}$ retention volume, and the low-molecular-weight fraction comprehensive of degraded chains (oligomers, monomers, etc.), named peak 2, ranging from 17.5 to 19 min retention volume (see Supplementary Figure S1). It is important to underline that the values reported (Mw, Mn and IV) must be attributed to a mix of undegradable POLOX and PLGA. 


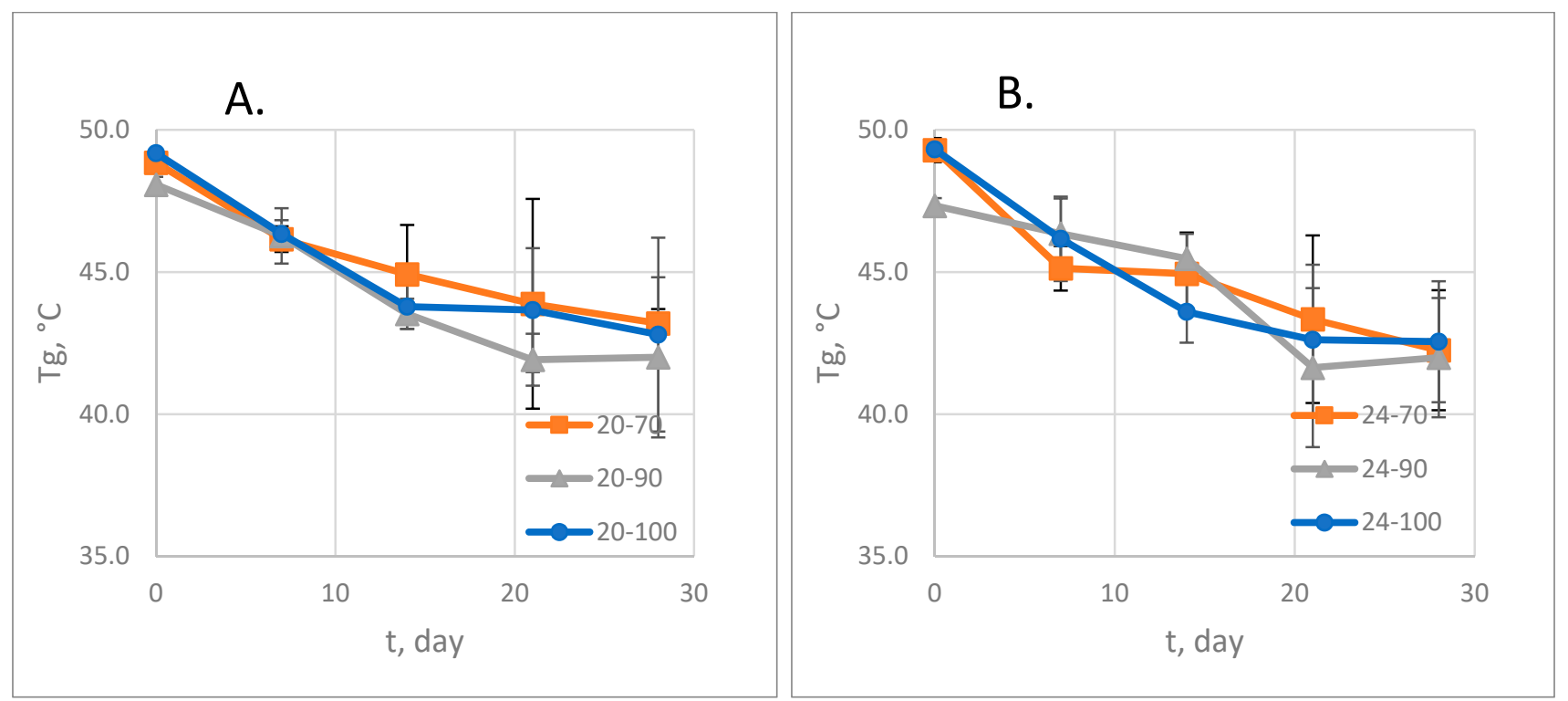

Figure 4. Trends of PLGA glass transition temperatures. (A): 20—70, 20—90, 20—100 formulations; (B): 24-70, 24—90, 24-100 formulations.

Comparing the 20-100 and 24-100 series, it is possible to note that the molecular weights and IV decreased over time, with the same trend (Figure 5), as expected due to the similar porous appearance of MPs (see Figure 1). Instead, the ratio between peak 1 and peak 2 was different, possibly due to the large amount of degraded PLGA (entries 4 and 16 in Supplementary Table S1).

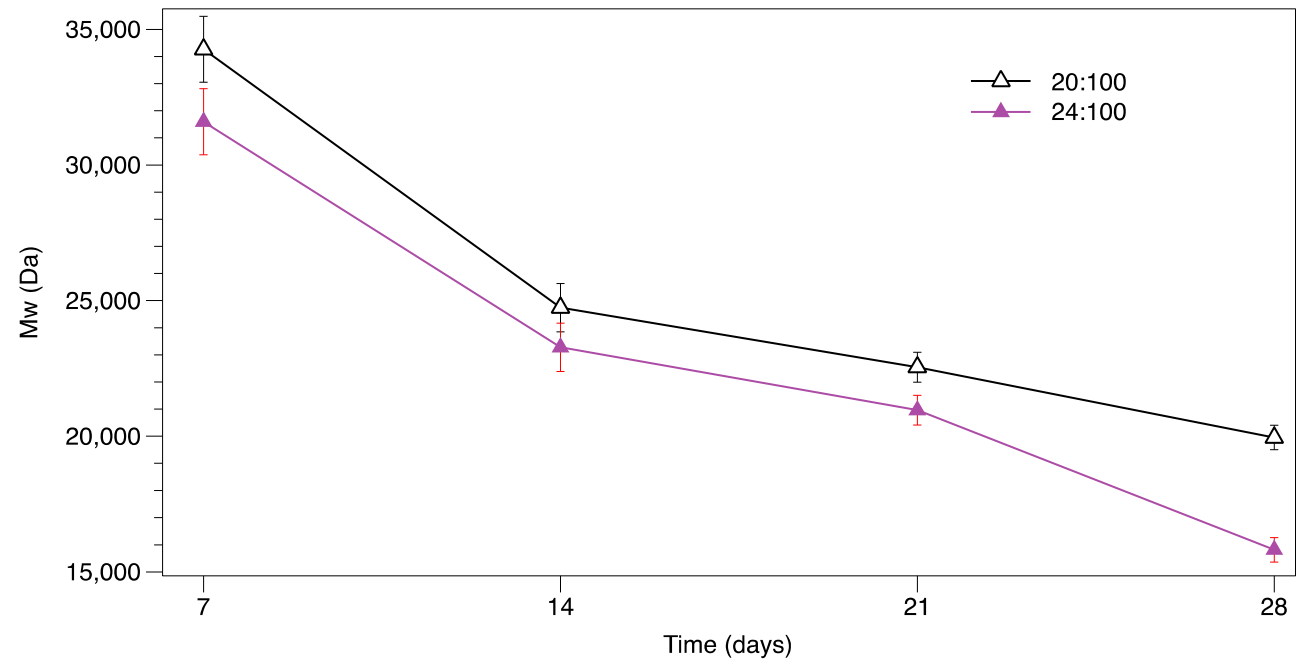

Figure 5. Time trend of for 20-100 and 24-100 (PLGA—only) MPs.

In any series, except 24:100, after 14 days, PLGA decomposed significantly slower, in line with the release kinetics and initial release data (Figures 2 and 3), therefore indicating an erosion-driven release mechanism.

In the 20:90 series, it is possible to note that $\mathrm{Mw}$ was halved at the final point and, at the same time point, IV was higher than in previous samples, with a value close to pure POLOX F127. This could be explained by the fact that peak 1 (9-15 min retention volume) was composed mainly of POLOX as consequence of the "high" degradation rate of PLGA. This may indicate that POLOX cannot exert a satisfactory "shielding effect" due to their low amount in the 20-90 formulation. This hypothesis is supported by the values of peak 1 and peak 2. 
In the 20:70 series, the higher concentration of POLOX led to a "slower" degradation, as indicated by peaks 1 and 2; however, at the final point IV slightly increased outside of the trend, showing unsatisfactory protection of PLGA from degradation.

In the 24:90 and 24:70 series, the higher concentration of polymers led to lower molecular weights at the starting points. In both series, and more evidently in the 24:70 series, it is possible to see (Figure 6) the presence of POLOX in peak 1 at 14,21 and 28 days, and at the last point, it is possible to note a shoulder at $10.3 \mathrm{~min}$ attributable to un-degraded PLGA.

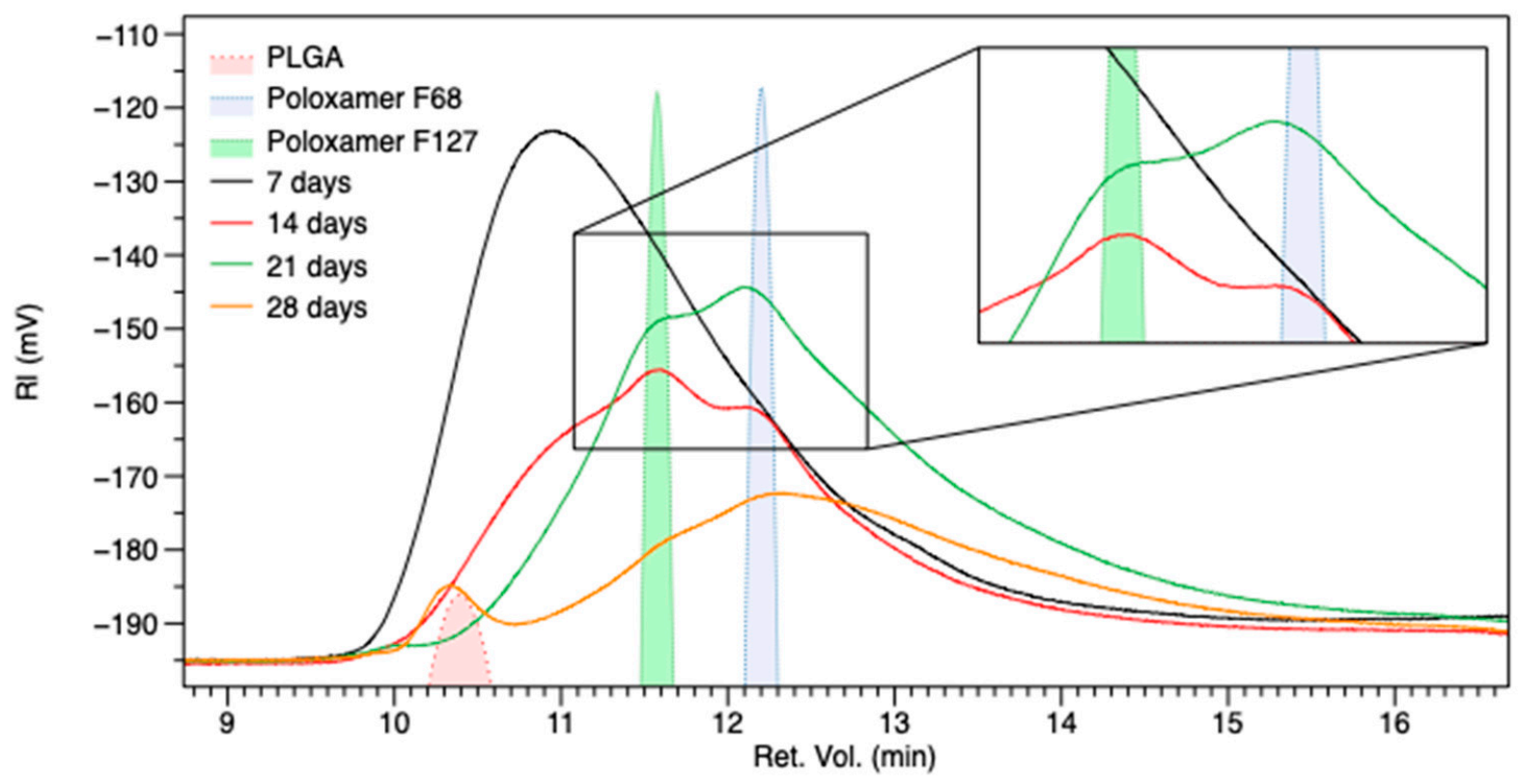

Figure 6. Superimposed chromatograms at RI detector of 24:70 series and in full red pure PLGA, in full green F127 POLOX, in full blue F68 POLOX. Chromatograms from 14 days show the presence of poloxamers and, at 28 days, a residual peak of PLGA.

Overall, the results indicate that the formulation conditions had a relatively weak effect on the MP degradation kinetics. More specifically, the decrease in overall MP molecular weight in the absence of POLOX was faster for the 24-100 compared to the 20-100 formulations. This indicates that, despite the porous morphology of microdevices, autocatalysis is enhanced at the highest polymer concentration. Moreover, 20-90 and 24-90 MPs showed a slightly increased degradation rate, to a higher extent in the case of the 24-90 formulation. This can be explained considering that POLOX facilitate water penetration, with autocatalysis being very likely due to the high polymer concentration. Consequently, the formation of short PLGA chains in the MP bulk is promoted by the enhanced water penetration, while the transport of degradation products is hampered. At the same time, the increased MP hydrophilicity is expected to facilitate base penetration in MPs, which neutralizes the generated acids. The overall balance suggests that acid generation is probably faster than the neutralization rate $[25,26]$, which is related to a drop in local $\mathrm{pH}$ within MPs.

The presence of a higher POLOX concentration in 20-70 and 24-70 MPs, on the contrary, induced a degradation rate closer to that of 20-100 and 24-100 MPs. In fact, an increasing POLOX amount is expected to promote water intrusion in MPs, and this causes the enhanced mobilization of the acidic degradation products of PLGA. Accordingly, the decrease in $\mathrm{pH}$ in the MP bulk is probably less marked, resulting in slightly inhibited autocatalysis compared to the 20-90 and 24-90 formulations. This mildly impacts the BSA release profiles (Figure 2). It must be underlined that BSA mobilization is dictated by the complex hydrophilicity/hydrophobicity interplay, which is, in turn, affected by the changing PLGA molecular weight and POLOX solubilization. Indeed, a lower molecular weight of PLGA results in the promotion of BSA transport and increased polymer hy- 
drophilicity due to an increased number of carboxylic groups per mass unit. The increased hydrophilicity associated with higher POLOX weight ratios has different and conflicting consequences: (i) it causes an enhancement in BSA mobility; (ii) MP erosion is promoted by the leaking of degradation products and soluble moieties in the surrounding gel, leading to the formation of new pores; (iii) autocatalysis is discouraged due to the loss of soluble acids. All in all, POLOX in MP inner regions induces different overall effects on BSA discharge, which are also affected by the total polymer concentration.

\section{Conclusions}

Interestingly, PLGA blending with POLOX for the production of MPs for intravitreal release offers the potential to adjust the protein release profiles by manipulating the mass ratio between PLGA and POLOX. More specifically, POLOX caused a compact MP matrix, which possibly affected the water penetration in the MP bulk in a complex manner. This led to a slight modification of the PLGA MP degradation kinetics. The reduction in initial release is of the utmost importance to ensure relatively regular BSA discharge and, in this context, the amount of POLOX is a pivotal design parameter. It must also be underlined that for acid-labile molecules such as proteins, POLOX's presence induced complex and hardly predictable effects on MP degradation/protein release, due to the dynamic balance between the time-evolving hydrophilicity of MPs and the influence of POLOX themselves on the PLGA degradation rate. Future studies will be needed to achieve a comprehensive understanding of this balance, which is necessary for the rational tailoring of protein release from biodegradable MPs intended for intravitreal administration. An important caveat to keep in mind is that the rate of protein release and device disappearance must match in optimized formulations. Indeed, this is of extreme importance in the view of intravitreal administration, for which a strong requirement is the absence of MP debris after each injection.

Supplementary Materials: The following are available online at https:/ / www.mdpi.com/article/10 .3390/app11167567/s1, Figure S1: Representative GPC chromatogram at RI detector showing peak 1 and peak 2, Table S1: Weight-average molecular weight $(\mathrm{Mw})$, number-average molecular weight (Mn), intrinsic viscosity (IV) and weight fraction of peak1 and peak2 as determined by GPC.

Author Contributions: Conceptualization, L.M. and M.B.; methodology, L.M., M.B., P.D.D., T.S. and B.I.; data curation, V.M., T.S. and G.D.P.; writing-original draft preparation, all authors; writing-review and editing, L.M. and M.B.; supervision, L.M. and M.B.; project administration, L.M. and M.B.; funding acquisition, L.M. and M.B. All authors have read and agreed to the published version of the manuscript.

Funding: This research was funded by P.O.R. CAMPANIA FSE 2014/2020-ASSE III-OBIETTIVO SPECIFICO 14 Azione 10.4.5.

Acknowledgments: The authors warmly thank Paolo Antonio Netti for providing the SEM apparatus.

Conflicts of Interest: The authors declare no conflict of interest.

\section{References}

1. Khalil, H.E.D.M.; El Gendy, H.A.; Youssef, H.A.R.; Haroun, H.E.; Gheita, T.; Bakir, H.M. The Effectiveness of Intraocular Methotrexate in the Treatment of Posterior Uveitis in Behçet's Disease Patients Compared to Retrobulbar Steroids Injection. J. Ophthalmol. 2016, 2016, 1-5. [CrossRef] [PubMed]

2. Herrero-Vanrell, R.; Refojo, M.F. Biodegradable microspheres for vitreoretinal drug delivery. Adv. Drug Deliv. Rev. 2001, 52, 5-16. [CrossRef]

3. Geroski, D.H.; Edelhauser, H.F. Drug delivery for posterior segment eye disease. Investig. Ophthalmol. Vis. Sci. 2000, 41, 961-964.

4. Edelhauser, H.F.; Rowe-Rendleman, C.L.; Robinson, M.R.; Dawson, D.G.; Chader, G.J.; Grossniklaus, H.E.; Rittenhouse, K.D.; Wilson, C.G.; Weber, D.A.; Kuppermann, B.D.; et al. Ophthalmic Drug Delivery Systems for the Treatment of Retinal Diseases: Basic Research to Clinical Applications. Investig. Opthalmol. Vis. Sci. 2010, 51, 5403-5420. [CrossRef] [PubMed]

5. Bodor, N.; Buchwald, P. Ophthalmic drug design based on the metabolic activity of the eye: Soft drugs and chemical delivery systems. AAPS J. 2005, 7, E820-E833. [CrossRef] [PubMed]

6. Gaudana, R.; Ananthula, H.K.; Parenky, A.; Mitra, A.K. Ocular Drug Delivery. AAPS J. 2010, 12, 348-360. [CrossRef] 
7. Falavarjani, K.G.; Nguyen, Q.D. Adverse events and complications associated with intravitreal injection of anti-VEGF agents: A review of literature. Eye 2013, 27, 787-794. [CrossRef] [PubMed]

8. Herrero-Vanrell, R.; Ramirez, L.; Fernandez-Carballido, A.; Refojo, M.F. Biodegradable PLGA Microspheres Loaded with Ganciclovir for Intraocular Administration. Encapsulation Technique, in Vitro Release Profiles, and Sterilization Process. Pharm. Res. 2000, 17, 1323-1328. [CrossRef] [PubMed]

9. Janoria, K.G.; Gunda, S.; Boddu, S.H.; Mitra, A.K. Novel approaches to retinal drug delivery. Expert Opin. Drug Deliv. 2007, 4, 371-388. [CrossRef]

10. Sampat, K.M.; Garg, S.J. Complications of intravitreal injections. Curr. Opin. Ophthalmol. 2010, 21, 178-183. [CrossRef]

11. Joseph, M.; Trinh, H.M.; Cholkar, K.; Pal, D.; Mitra, A.K. Recent perspectives on the delivery of biologics to back of the eye. Expert Opin. Drug Deliv. 2016, 14, 631-645. [CrossRef]

12. Serri, C.; Frigione, M.; Ruponen, M.; Urtti, A.; Borzacchiello, A.; Biondi, M.; Itkonen, J.; Mayol, L. Electron dispersive X-ray spectroscopy and degradation properties of hyaluronic acid decorated microparticles. Colloids Surf. B Biointerfaces 2019, 181, 896-901. [CrossRef]

13. Mayol, L.; Silvestri, T.; Fusco, S.; Borzacchiello, A.; De Rosa, G.; Biondi, M. Drug micro-carriers with a hyaluronic acid corona toward a diffusion-limited aggregation within the vitreous body. Carbohydr. Polym. 2019, 220, 185-190. [CrossRef]

14. Giarra, S.; Serri, C.; Russo, L.; Zeppetelli, S.; de Rosa, G.; Borzacchiello, A.; Biondi, M.; Ambrosio, L.; Mayol, L. Spontaneous arrangement of a tumor targeting hyaluronic acid shell on irinotecan loaded PLGA nanoparticles. Carbohydr. Polym. 2016, 140. [CrossRef]

15. Kogan, G.; Šoltés, L.; Stern, R.; Gemeiner, P. Hyaluronic acid: A natural biopolymer with a broad range of biomedical and industrial applications. Biotechnol. Lett. 2006, 29, 17-25. [CrossRef] [PubMed]

16. Netti, P.A.; Biondi, M.; Frigione, M. Experimental Studies and Modeling of the Degradation Process of Poly(Lactic-co-Glycolic Acid) Microspheres for Sustained Protein Release. Polymers 2020, 12, 2042. [CrossRef] [PubMed]

17. Biondi, M.; Indolfi, L.; Ungaro, F.; Quaglia, F.; La Rotonda, M.I.; Netti, P.A. Bioactivated collagen-based scaffolds embedding protein-releasing biodegradable microspheres: Tuning of protein release kinetics. J. Mater. Sci. Mater. Med. 2009, 20, $2117-2128$. [CrossRef] [PubMed]

18. Ding, A.G.; Schwendeman, S.P. Determination of water-soluble acid distribution in poly(lactide-co-glycolide). J. Pharm. Sci. 2004, 93, 322-331. [CrossRef]

19. Siepmann, J.; Elkharraz, K.; Siepmann, A.F.; Klose, D. How Autocatalysis Accelerates Drug Release from PLGA-Based Microparticles: A Quantitative Treatment. Biomacromolecules 2005, 6, 2312-2319. [CrossRef]

20. Schädlich, A.; Kempe, S.; Mäder, K. Non-invasive in vivo characterization of microclimate pH inside in situ forming PLGA implants using multispectral fluorescence imaging. J. Control Release 2014, 179, 52-62. [CrossRef]

21. Kummer, M.P.; Abbott, J.J.; Dinser, S.; Nelson, B.J. Artificial vitreous humor for in vitro experiments. In Proceedings of the 2007 29th Annual International Conference of the IEEE Engineering in Medicine and Biology Society, Lyon, France, $22-26$ August 2007. [CrossRef]

22. Corrigan, O.I.; Li, X. Quantifying drug release from PLGA nanoparticulates. Eur. J. Pharm. Sci. 2009, 37, 477-485. [CrossRef]

23. Batycky, R.P.; Hanes, J.; Langer, R.; Edwards, D.A. A Theoretical Model of Erosion and Macromolecular Drug Release from Biodegrading Microspheres. J. Pharm. Sci. 1997, 86, 1464-1477. [CrossRef] [PubMed]

24. Patel, R.R.; Chaurasia, S.; Khan, G.; Chaubey, P.; Kumar, N.; Mishra, B. Cromolyn sodium encapsulated PLGA nanoparticles: An attempt to improve intestinal permeation. Int. J. Biol. Macromol. 2016, 83, 249-258. [CrossRef] [PubMed]

25. Siepmann, J. Mathematical modeling of bioerodible, polymeric drug delivery systems. Adv. Drug Deliv. Rev. 2001, 48, 229-247. [CrossRef]

26. Von Burkersroda, F.; Schedl, L.; Göpferich, A. Why degradable polymers undergo surface erosion or bulk erosion. Biomaterials 2002, 23, 4221-4231. [CrossRef] 